\title{
Factors Associated with COVID-Related Mortality: the Case of Texas
}

\author{
Chinedum O. Ojinnaka ${ }^{1} \cdot$ Omolola E. Adepoju ${ }^{2,3}$ (D) Anh Vi Burgess ${ }^{4} \cdot$ LeChauncy Woodard $^{2,3}$
}

Received: 2 July 2020 / Revised: 12 October 2020 / Accepted: 27 October 2020 / Published online: 9 November 2020

(C) This is a U.S. government work and its text is not subject to copyright protection in the United States; however, its text may be subject to foreign copyright protection 2020

\begin{abstract}
Background Texas ranks 2nd in the count of COVID cases. Pre-existing disparities in healthcare may be intersecting with COVID-19 outcomes.

Objectives To explore the relationship between county-level race/ethnic composition and COVID-19 mortality in the state of Texas and determine whether county-level health factors, healthcare access measures, and other demographic characteristics explain this relationship.

Methods This retrospective study uses county-level case and fatality data obtained from the Texas Department of State Health Services and merged with the 2020 Robert Wood Johnson foundation (RWJF) county health rankings data. The outcome variables were fatalities per 100,000 population. A two-part/hurdle model examined (1) the probability of having a COVID19 fatality and (2) fatalities per 100,000 population in counties with 1+ fatalities. For both parts of the hurdle model, we examined the impacts of racial and ethnic composition, adjusting for county characteristics and health factors.

Results The odds of having a COVID-19 fatality decreased with a unit increase in the rate of primary care physicians in a county $(\mathrm{OR}=0.93 ; 95 \% \mathrm{CI}=0.89,0.99)$. In the second part of the model, there was a statistically significant increase in COVID-19 fatalities $/ 100,000$ population with every $1 \%$ increase in the proportion of Hispanics ( $\beta=5.41 ; p=0.03)$ and African Americans $(\beta=5.08 ; p$ value $=0.04)$.

Conclusion Counties with higher rates of minorities, specifically Hispanics and African Americans, have a higher COVID-19 fatality burden. Targeted interventions are needed to raise awareness of preventive measures in these communities.
\end{abstract}

Keywords COVID-19 mortality $\cdot$ Racial disparities $\cdot$ Healthcare access $\cdot$ County-level factors

\section{Introduction}

The COVID-19 pandemic is by far the largest global health emergency in modern history. Over 7.6 million cases have been reported in the United States (US) resulting in excess of 200,000 deaths [1]. In addition to the sheer scale of the pandemic which has shown very slow signs of abatement, there are currently no

Omolola E. Adepoju

oadepoju@uh.edu

1 College of Health Solutions, Arizona State University, Phoenix, AZ, USA

2 Humana Integrated Health System Sciences Institute, University of Houston, Houston, TX, USA

3 Department of Health Systems and Population Health Sciences, University of Houston College of Medicine, 4849 Calhoun Road, Bldg 2, Houston, TX 77204, USA

4 Rice University, Houston, TX, USA approved cures or vaccines for COVID-19. State and federal governments have instituted stay-at-home mandates, handwashing, social distancing, and respiratory hygiene to slow the spread of the virus. Early findings suggest that being male, age 50 and older, and having one or more underlying medical conditions are associated with a COVID-19 hospitalization.

COVID-19 affects different states and cities in the USA at varying intensities and timeframes. As of September 2020, Texas ranked 2nd in the number of total cases. The numbers of cases in Texas reflect an upward trend throughout the months of May, June, and July, and coincide with the expiration of statewide stay-at-home orders [2, 3]. COVID-related hospitalizations have also increased during this time period, reaching over 10,000 hospitalized cases in a single day in July [3]. Hospitals in the Texas Medical Center, the largest medical center in the world, have had to invoke surge planning as ICU capacity exceeded 100\% [4]. Descriptive reporting suggests that the majority of the COVID-19 cases have occurred in 20 59 year olds; however, nearly a third of the deaths reported thus far have occurred individuals 80 and older [3]. The five 
counties with the most fatalities are home to some major cities including Dallas, Houston, and San Antonio but two counties at the Texas-Mexico border in the Rio Grande Valley have also reported high fatality rates [3]. Although the concentration of fatalities is concerning, Harris, Bexar, and Dallas Counties have increased hospital capacities, unlike some rural counties, where there are fewer per capita cases but the cases are increasing at a greater rate than metropolitan areas [5].

Many have suggested that prior existing disparities in healthcare, based on race, ethnicity, and rurality, may be intersecting with the COVID-19 pandemic to worsen the disease burden in vulnerable populations. Long-standing systemic inequities that people of color face have allowed for racial disparities to become magnified in the setting of this public health crisis. Recent racial data has revealed that COVID-19 is disproportionately impacting communities of color across the USA. Black Americans suffer a devastating mortality rate to COVID-19, at approximately 3.4 times as high as the mortality rate for their white counterparts. Although they only represent about $12.4 \%$ of the US population, Black Americans make up over $20 \%$ of reported deaths related to COVID-19 [6]. In addition, Hispanic Americans are disproportionately testing positive for COVID-19 across most of the nation [7]. In Texas specifically, nearly $40 \%$ of cases and $56 \%$ of deaths have occurred in the Hispanic population; in the African American population in Texas, COVID-19 cases and fatality distribution are 16 and $11 \%$, respectively [3].

While these numbers are disturbing, it unfortunately is no surprise that racial minority groups bear the greatest burden of disease. Racial minorities make up approximately $41.2 \%$ of frontline workers in the United States, putting them at a significantly greater risk for COVID-19 exposure and subsequent infection than Americans who have the comfort of working from home [8]. Additionally, people of color face higher rates of unemployment and underemployment than white workers [9]. Thus, racial minorities may be more likely to take temporary occupational opportunities that may put them at a greater risk for COVID-19 exposure, like food delivery. Lower socioeconomic status due to unemployment or underemployment may limit the degree to which an individual can minimize their exposure to COVID-19 and socially distance from others. For example, low-income families that qualify for SNAP benefits in the USA have not been able to purchase their groceries online for delivery until recently, and even then, the list and locations of grocery stores that allow for this service is limited. Instead, some may have no choice but to enter a busy grocery store, thereby putting them at a greater risk for COVID-19 exposure.

In addition, chronic diseases, which are associated with increased severity and mortality in COVID-19, tend to be overrepresented in communities of color. Black and Hispanic Americans are disproportionately affected by chronic disease burden [10]. More specifically, multiple underlying chronic conditions, including type II diabetes, obesity, and hypertension, are significantly more prevalent in Black Americans than their white counterparts [11]. This disparity is further perpetuated by the gaps that exist in chronic disease management. A notable contribution to this gap is the fact that minorities are more likely to report higher levels of physician distrust [12], due in part to the deep history of racism in the practice of medicine that continues in the health care system today. Unethical research and experimentation on Black individuals, including but certainly not limited to the Tuskegee syphilis experiment, Henrietta Lacks' "immortal cells," and James Marion Sims' surgical experiments, have long exploited Black bodies to bring about medical advancement. The modern-day lack of trust in physicians by Black Americans is complex, but nevertheless relates to the lack of willingness to seek health care when they need it and contributes to the persisted burden of chronic disease.

The interplay between these sociodemographic factors demonstrates a need for population-level studies to evaluate determinants of COVID-19 fatality and to develop policies and strategies to prevent excess mortality in populations at greatest risk. To our knowledge, no study has analyzed the intersection of race/ethnicity, primary care availability and other health characteristics in relation to Texas COVID-19 deaths. This paper uses county-level COVID-19 fatality data to (1) explore the relationship between county-level race/ethnic composition and COVID-19 fatality in the state of Texas and (2) determine whether county-level health factors, healthcare access measures, and other demographic characteristics explain this relationship. Findings from this study are especially important as Texas and other states have been forced to pause reopening due to burgeoning COVID-19 cases subsequent to the relaxation of stay-at-home mandates.

\section{Methods}

\section{Data}

Publicly available Texas COVID-19 case and fatality data (from March 04, 2020 when the first Texas case was detected, through August 1, 2020) were obtained from the Texas Department of State Health Services Center for Health Statistics COVID-19 Dashboard. Fatality Data on all 254 Texas counties are reported by date. This dataset was merged with the 2020 Robert Wood Johnson Foundation (RWJF) County Health Rankings data. The RWJF's County Health Ranking datasets contain information on demographic characteristics, economic, environmental, and health-related factors to provide a better understanding of community context. Importantly, they provide county-level indicators of health behaviors, healthcare outcomes, and factors that impact overall health. Because data was obtained from publicly available 
sources, research procedures fit within one or more of the exemption categories in the federal institutional review board (IRB) regulations.

\section{Measures}

There were two outcomes of interest: (1) COVID-19 death (no (0), yes [1]) and (2) cumulative COVID-19 fatalities per 100,000 population in counties with one or more COVID-19 deaths. The independent variables of interest were county-level racial/ethnic composition, reported as percent non-Hispanic White, Black, American Indian/ Alaska Native, Asian, Native Hawaiian/Other Pacific Islander and Hispanic. We controlled for health factors (tobacco use, obesity, diabetes, fair or poor health), healthcare access measures (insurance status and primary care physician rate), and other county-level demographics (high school graduation rate, unemployment, black-white segregation index and rurality).

\section{Statistical Analyses}

Univariate analyses were used to report county-level economic, environmental, and health-related characteristics. Because $20 \%$ of counties reported zero fatalities, a twopart/hurdle model was used to (1) examine the probability of a COVID-19 related mortality and (2) assess determinants of fatalities per 100,000 population in counties with one or more fatalities. For both parts of the hurdle model, we examined the association between our outcome of interest and county-level race/ethnic composition controlling for health factors, healthcare access measures and other county-level demographic characteristics. A total of four models were estimated as follows: (i) logistic regression model with COVID-19 related fatality $(0 / 1)$ as the dependent variable, and proportion of racial/ethnic groups as the only independent variables; (ii) logistic regression model with COVID-19 related fatality $(0 / 1)$ as the dependent variable, and proportion of racial/ethnic groups + healthcare access measures + other demographic characteristics as the independent variables; (iii) generalized linear models with fatalities per 100,000 population as the dependent variable and proportion of racial/ethnic groups alone as the only independent variables; and (iv) generalized linear models with fatalities per 100,000 population as the dependent variable and proportion of racial/ethnic groups + healthcare access measures + other demographic characteristics as the independent variables. Sensitivity analyses were performed using robust linear regression. All statistical tests were 2 -sided, and findings were considered statistically significant at $P<.05$. All analyses were conducted using Stata 15.1.

\section{Results}

Table 1 shows descriptive statistics of the independent variables. The percentage of non-Hispanic Whites ranged from 2.69 to $89.4($ mean $=55.34$; std . dev. $=21.04)$, African Americans/Blacks ranged from 0 to 33.14 (mean =6.26; std. dev. $=6.40)$, and Asians ranged from 0 to 20.79 (mean = 1.33; std. dev. =2.08). The percentage of Hispanics ranged from 3.72 to $96.36($ mean $=35.35$; std. dev. $=22.99)$. The percentage of smokers ranged from 10.64 to 19.87 (mean $=14.94$; std. dev. $=1.56$ ). Primary care physician rate ranged from 0 to 141.50 (mean $=40.50$; std. dev. 25.85).

Table 2 shows the unadjusted and adjusted odds of a COVID-19 fatality. In the unadjusted model, the likelihood of a COVID-19 death increased with increasing proportion of Asians $(\mathrm{OR}=7.53 ; 95 \% \mathrm{CI}=1.99,28.36)$ and African Americans $(\mathrm{OR}=1.95 ; 95 \% \mathrm{CI}=1.03,3.68)$. After controlling for health factors, healthcare access measures, and other county-level demographic characteristics, this race/ethnicity association was no longer significant. However, there was a decreased likelihood of COVID-19 death with a unit increase in the rate of primary care physicians in the county $(\mathrm{OR}=0.93 ; 95 \% \mathrm{CI}=0.89,0.99)$.

Table 3 shows multivariable analyses of COVID-19 fatality/100,000 in counties with at least one COVID-19 death. In the unadjusted model, there was a statistically significant increase in COVID-19 deaths/100,000 population with every 1 $\%$ increase in the proportion of African Americans/Blacks ( $\beta=6.04 ; 95 \% \mathrm{CI}=0.94,11.14)$, non-Hispanic Whites $(\beta=$ $5.38 ; 95 \% \mathrm{CI}=0.21,10.34)$, and Hispanics $(\beta=5.63 ; 95 \%$ $\mathrm{CI}=0.67,10.59)$. In the final adjusted model, we observed a significant increase in COVID-19 deaths with every $1 \%$

Table 1 Summary STATISTICS OF COUNTY-LEVEL CHARACTERISTICS

\begin{tabular}{lrrrr}
\hline Variable & Min & Max & Mean & Std. Dev. \\
\hline Percent non-Hispanic white & 2.69 & 89.40 & 55.39 & 21.04 \\
Percent Black & 0.00 & 33.14 & 6.26 & 6.40 \\
Percent Asian & 0.00 & 20.79 & 1.33 & 2.08 \\
Percent Hispanic & 3.72 & 96.36 & 35.35 & 22.99 \\
Percent smokers & 10.64 & 19.87 & 14.94 & 1.56 \\
Percent adults with obesity & 21.60 & 47.30 & 31.25 & 5.26 \\
Percent adults with diabetes & 3.50 & 29.30 & 11.36 & 4.62 \\
Percent fair or poor health & 12.29 & 40.99 & 20.60 & 4.94 \\
Percent uninsured & 11.40 & 33.75 & 21.21 & 4.05 \\
Primary care physician rate & 0.00 & 141.50 & 40.50 & 25.85 \\
High school graduation rate & 61.80 & 100.00 & 93.65 & 5.67 \\
Percent unemployed & 1.83 & 10.10 & 3.87 & 1.26 \\
Black White Segregation Index & 1.67 & 76.78 & 39.98 & 14.03 \\
Percent Rural & 0.69 & 100.00 & 55.52 & 31.90 \\
\hline
\end{tabular}


Table 2 Logistic regression analyses of probability of county-level COVID-19 fatality

\begin{tabular}{lll}
\hline & $\begin{array}{l}\text { OR }(95 \% \text { CI }) \\
\text { Model 1 }\end{array}$ & $\begin{array}{l}\text { OR }(95 \% \text { CI }) \\
\text { Model } 2\end{array}$ \\
\hline Percent Black & $\mathbf{1 . 9 5 ( 1 . 0 3 , \mathbf { 3 . 6 8 } )}$ & $0.83(0.15,4.49)$ \\
Percent Non-Hispanic White & $1.61(0.87,2.99)$ & $0.64(0.10,4.15)$ \\
Percent Asian & $\mathbf{7 . 5 3 ( 1 . 9 9 , \mathbf { 2 8 . 3 6 } )}$ & $0.71(0.04,12.45)$ \\
Percent Hispanic & $1.65(0.90,3.02)$ & $0.67(0.11,3.85)$ \\
Percent Smokers & & $0.12(0.01,1.31)$ \\
Percent adults with obesity & & $1.18(0.88,1.59)$ \\
Percent adults with diabetes & & $0.94(0.74,1.80)$ \\
Percent fair or poor health & & $1.18(0.26,5.36)$ \\
Percent uninsured & & $1.01(0.61,1.70)$ \\
Primary care physician rate & & $\mathbf{0 . 9 4}(\mathbf{0 . 8 9}, \mathbf{1 . 0 0})$ \\
High school graduation rate & & $0.84(0.53,1.32)$ \\
Percent unemployed & & $0.97(0.26,3.62)$ \\
Black White segregation index & & $1.00(0.93,1.08)$ \\
Percent rural & & $0.92(0.85,1.00)$ \\
Percent 65 years or older & & $1.04(0.61,1.78)$ \\
\hline
\end{tabular}

increase in the proportion of African Americans/Blacks $(\beta=$ 5.08; $95 \% \mathrm{CI}=0.11,10.04)$ and Hispanics $(\beta=5.41 ; 95 \%$ $\mathrm{CI}=0.52,10.31$, but not for non-Hispanic whites or Asians. In addition, there was a significant increase in COVID-19 deaths $/ 100,000$ with every $1 \%$ increase in the proportion of adults with obesity $(\beta=0.71 ; 95 \% \mathrm{CI}=0.12,1.30)$, unemployed individuals $(\beta=5.21 ; 95 \% \mathrm{CI}=2.22,8.20)$ and in primary care physician rate $(\beta=0.17 ; 95 \% \mathrm{CI}=0.02,0.32)$.

\section{Discussion}

In this study, we examined disparities in COVID-19-related mortality, adjusting for demographic characteristics and health-related factors in Texas. To date, few studies have examined associations between county demographics, health characteristics, and COVID-19 fatalities in Texas. Our findings suggest that while COVID-19 might be an equal opportunity disease, counties with higher proportions of African Americans and Hispanics experience significantly larger mortality rates in Texas. These findings align with other racial/ ethnic reports on COVID-19 morbidity and mortality that suggest minorities, including African Americans, Hispanics, American Indian, Alaska Native, and Pacific Islander populations bear a disproportionate burden of disease [13]. Mahajan \& Larkins-Pettigrew (2020), in their analysis of all US states, observed a weak but significant correlation between African American race and COVID-19 related mortality, indicating that counties with a higher proportion of African Americans have higher COVID-19 fatalities [14].

Several underlying factors could contribute to the greater burden of COVID-19 fatality among minority populations. African Americans and Hispanics face higher rates of chronic disease prevalence and report poorer outcomes on several health indicators, compared with their non-Hispanic white counterparts [15]. Importantly, before the COVID-19 pandemic, there were major health disparities ingrained into the system that disproportionately impacted minority populations, making them more prone to chronic diseases. Chronic diseases such as diabetes, heart disease, and obesity occur mainly
Table 3 Multivariable regression analyses of COVID-19 deaths for counties with at least one death

\begin{tabular}{llc}
\hline & \multicolumn{2}{l}{ Robust Regression } \\
\cline { 2 - 3 } & $\begin{array}{l}\text { Model 3 } \\
\text { Coef. }(95 \% \mathrm{CI})\end{array}$ \\
\hline Percent Black & $6.04(0.94,11.14)$ & $\begin{array}{l}\text { Model 4 } \\
\text { Coef. }(95 \% \mathrm{CI})\end{array}$ \\
Percent Non-Hispanic White & $5.38(0.21,10.34)$ & $5.08(0.11,10.04)$ \\
Percent Asian & $4.67(-0.73,10.07)$ & $4.78(-0.28,9.83)$ \\
Percent Hispanic & $5.63(0.67,10.59)$ & $5.17(-0.11,10.45)$ \\
Percent Smokers & & $5.41(0.52,10.31)$ \\
Percent adults with obesity & & $0.99(-3.65,5.63)$ \\
Percent adults with diabetes & & $0.71(0.12,1.30)$ \\
Percent fair or poor health & & $-0.04(-0.66,0.59)$ \\
Percent uninsured & & $-0.71(-3.80,2.38)$ \\
Primary care physician rate & & $-0.27(-1.26,0.73)$ \\
High school graduation rate & & $0.17(0.02,0.32)$ \\
Percent unemployed & & $-0.07(-0.65,0.51)$ \\
Black White Segregation Index & & $5.21(2.22,8.20)$ \\
Percent rural & & $0.18(-0.02,0.38)$ \\
Percent 65 years or older & & $0.13(-0.04,0.31)$ \\
\hline
\end{tabular}


because of lifestyle and environment factors [16]. However, one's lifestyle is not always created by choice. Many of these individuals work multiple jobs, leaving very little time or resources for them to prepare healthy meals at home, exercise daily, and/or get ample sleep, along with all the other obligations they may have. Many low-income neighborhoods also exist in areas without easy access to grocery stores that provide healthy eating options or walkable neighborhoods [17]. In addition, African Americans are more likely live in densely populated areas where social distancing practices can be difficult to implement [18], making them more likely to contract the disease. Unfortunately, these inequities driven by social determinants often result in healthcare being relegated a lower priority as more immediate concerns demand attention. These factors contributing to increased prevalence of chronic diseases in African American communities are then further exacerbated by a lack of trust in health care that exists due to historic injustices suffered by these populations.

Our finding on Hispanic Americans' aligns with other reports that have reported increased mortality in this population $[19,20]$. These reports suggest that the increased mortality may be due to higher rates of comorbid medical conditions such as hypertension and diabetes and a high proportion of frontline workers [19, 21]. In addition, poorer access to health services due to immigration status, language barriers, and lack of convenient clinics and hospitals in minority neighborhoods may contribute to delays in seeking care which may lead to worse outcomes [19, 21]. Another systemic factor contributing to the disproportionate COVID-19 burden on the Hispanic community may be higher rates of uninsurance, which others have attributed to Texas' refusal to expand Medicaid. Currently, $10 \%$ of African Americans and $61 \%$ of Hispanics in Texas are uninsured, compared with $14 \%$ of African Americans and $37 \%$ of Hispanics nationwide [22]. Texas is home to approximately 1.6 million undocumented immigrants who comprise $6 \%$ of the population; none of those people have access to health insurance, which may have worsened outcomes in seeking care for COVID [23].

In addition to race/ethnicity disparities, our findings suggest that obesity portends a higher risk of developing and dying from COVID-19 [24]. This aligns with other studies that report obese individuals are more likely to develop severe symptoms and have worse COVID-19 outcomes, such as death [25-27]. We also observed an inverse relationship between the probability of COVID-19 fatality and rate of primary care physicians, aligning with earlier reports that Texas counties with case fatality rates greater than $10 \%$ also had the lowest number of primary care physicians per 100,000 [28]. Lastly, our findings suggest that increasing unemployment rates were associated with greater COVID-19 fatality, supporting earlier findings of increased incidence in counties with higher rates of poverty and unemployment [29]. Altogether, these findings suggest that COVID-related mortality is worse in communities with a large proportion of underserved populations, which is probably exacerbated by the sub-optimal healthcare access experienced by members of these communities.

This study is not without limitations. First, our analysis is at the county-level, using county-level aggregates of health factors ( $\%$ adults with diabetes, $\%$ adults with obesity, $\%$ smokers), healthcare access (\%uninsured, primary care physician rates), and other county-level demographic characteristics (\% racial/ethnic composition American, \% rural, \% unemployed, high school graduate rate, $\%$ unemployed). Findings using county aggregates may not generate similar findings if individual level characteristics are modeled. Notwithstanding, these findings highlight the need for targeted interventions to raise awareness of preventive measures in these communities most significantly impacted by COVID-19. Although it has been said that this virus "does not discriminate," the setting of a global pandemic has only exacerbated disparities that already exist in our healthcare systems, making them more obvious and heightening the urgency to identify interventions to reduce health inequity.

Authors' Contributions C. Ojinnaka and O. Adepoju contributed to the study conception and design. Material preparation, data collection, and analysis were performed by C. Ojinnaka and O. Adepoju. The first draft of the manuscript was written by A. Burgess. L. Woodard provided critical review for manuscript framing, and all authors commented on previous versions of the manuscript. All authors read and approved the final manuscript.

Data Availability Publicly available data.

\section{Compliance with Ethical Standards}

Conflict of Interest The authors have no conflicts of interest or competing interests to declare, financial or personal.

Code Availability Stata codes are available.

\section{References}

1. Prevention CfDCa. Cases in the U.S. 2020 [Available from: https:// www.cdc.gov/coronavirus/2019-ncov/cases-updates/cases-in-us. html. Accessed 9 Oct 2020.

2. Services TDoSH. Opening the State of Texas 2020 [Available from: https://www.dshs.texas.gov/coronavirus/opentexas.aspx. Accessed 30 Sept.

3. Texas Case Counts: COVID-19 [Internet]. 2020 [cited June 11, 2020]. Available from: https://txdshs.maps.arcgis.com/apps/ opsdashboard/index.html\#/ed483ecd702b4298ab01e8b9cafc8b83. Accessed 9 Oct 2020.

4. Texas Medical Center. Coronavirus (COVID-19) Updates. 2020 June 11, 2020.

5. Fehr R, Kates J, Cox C, Michaud J. COVID-19 in rural America - is there cause for concern? : Kaiser Family Foundation; 2020 June 10, 2020 . 
6. Lab AR. "The color of coronavirus: COVID-19 deaths by race and ethnicity in the US. 2020. https://www.apmresearchlab.org/covid/ deaths-byrace. Accessed 2 Jul 2020.

7. Racial Data Dashboard [Internet]. 2020 [cited July 1, 2020]. Available from: https://covidtracking.com/race/dashboard.

8. Rho JH, Brown H, Fremstad S. A basic demographic profile of workers in frontline industries. Cent Econ Pol Res. 2020. https:// cepr.net/a-basicdemographic-profile-of-workers-in-frontlineindustries/. Accessed 31 Jul 2020.

9. Shierholz H. "Roughly One in Five Hispanic and Black Workers Are 'Underemployed'. 2013. Washington, DC: Economic Policy Institute http://www.epi.org/publication/roughly-hispanic-blackworkers-underemployed. Accessed 5 Oct 2020.

10. Quiñones AR, Botoseneanu A, Markwardt S, Nagel CL, Newsom JT, Dorr DA, et al. Racial/ethnic differences in multimorbidity development and chronic disease accumulation for middle-aged adults. PLoS One. 2019;14(6):e0218462.

11. Price JH, Khubchandani J, McKinney M, Braun R. Racial/ethnic disparities in chronic diseases of youths and access to health care in the United States. Biomed Res Int. 2013;2013:1-12.

12. Armstrong K, Ravenell KL, McMurphy S, Putt M. Racial/ethnic differences in physician distrust in the United States. Am J Public Health. 2007;97(7):1283-9.

13. Webb Hooper M, Nápoles AM, Pérez-Stable EJ. COVID-19 and racial/ethnic disparities. Jama. 2020;323:2466-7.

14. Mahajan UV, Larkins-Pettigrew M. Racial demographics and COVID-19 confirmed cases and deaths: a correlational analysis of 2886 US counties. J Public Health (Oxf). 2020;42:445-7.

15. Cordner A, Wilkie AA, Wade TJ, Hudgens EE, Birch RJ, Gallagher JE. Gender and racial/ethnic disparities: cumulative screening of health risk indicators in 20-50 year olds in the United States. J Health Dispar Res Pract. 2017;10(8):1.

16. Kolb H, Martin S. Environmental/lifestyle factors in the pathogenesis and prevention of type 2 diabetes. BMC Med. 2017;15(1):131.

17. Child ST, Kaczynski AT, Fair ML, Stowe EW, Hughey SM, Boeckermann L, et al. 'We need a safe, walkable way to connect our sisters and brothers': a qualitative study of opportunities and challenges for neighborhood-based physical activity among residents of low-income African-American communities. Ethn Health. 2019;24(4):353-64.
18. Acevedo-Garcia D. Residential segregation and the epidemiology of infectious diseases. Soc Sci Med. 2000;51(8):1143-61.

19. Macias Gil R, Marcelin JR, Zuniga-Blanco B, Marquez C, Mathew T, Piggott DA. COVID-19 pandemic: disparate health impact on the Hispanic/Latinx population in the United States. J Infect Dis. 2020;222:1592-5.

20. Alcendor DJ. Racial disparities-associated COVID-19 mortality among minority populations in the US. J Clin Med. 2020;9(8). https://doi.org/10.3390/jcm9082442.

21. Selden TM, Berdahl TA. COVID-19 and racial/ethnic disparities in health risk, employment, and household composition. Health Aff (Millwood). 2020;39(9):1624-32.

22. Texas Medical Association. Quick statistics on the uninsured in Texas and the U.S.: Texas medical association; 2020. https:// www.texmed.org/template.aspx?id=5519. Accessed 19 Sept 2020.

23. American Immigration Council. Immigrants in Texas. American Immigration Council; 2020. https://americanimmigrationcouncil. org/research/immigrants-in-texas. Accessed 18 Sept 2020.

24. CDC COVID Response Team. Preliminary estimates of the prevalence of selected underlying health conditions among patients with coronavirus disease 2019 - United States, February 12-march 28, 2020. MMWR Morb Mortal Wkly Rep. 2020;69(13):382-6.

25. Zhou Y, Chi J, Lv W, Wang Y. Obesity and diabetes as high-risk factors for severe coronavirus disease 2019 (Covid-19). Diabetes Metab Res Rev. 2020:e3377. https://doi.org/10.1002/dmrr.3377.

26. Yang J, Hu J, Zhu C. Obesity aggravates COVID-19: a systematic review and meta-analysis. J Med Virol. 2020.

27. Hussain A, Mahawar K, Xia Z, Yang W, El-Hasani S. Obesity and mortality of COVID-19. Meta-analysis. Obes Res Clin Pract. 2020;14(4):295-300.

28. Khose S, Moore JX, Wang HE. Epidemiology of the 2020 pandemic of COVID-19 in the state of Texas: the first month of community spread. J Community Health. 2020;45(4):696-701.

29. Ramírez IJ, Lee J. COVID-19 Emergence and social and health determinants in colorado: a rapid spatial analysis. Int J Environ Res Public Health. 2020;17(11). https://doi.org/10.3390/ ijerph17113856.

Publisher's Note Springer Nature remains neutral with regard to jurisdictional claims in published maps and institutional affiliations. 